\title{
OPINIÓN
}

\section{Sugerencias para mejorar la calidad de las revistas científicas chilenas: una aplicación en las ciencias forestales con Bosque}

\author{
Suggestions to improve the quality of Chilean scientific journals: \\ an application to forest science with Bosque
}

\author{
Christian Salas ${ }^{\mathrm{a}, \mathrm{b}}$ \\ aUniversidad de La Frontera, Departamento de Ciencias Forestales, Temuco, Chile. \\ ${ }^{b}$ Yale University, School of Forestry and Environmental Studies, 360 Prospect Street, New Haven, CT 06511, USA, \\ tel.: 1(203)432-9398, fax: 1(203)432-3809, christian.salas@yale.edu
}

\begin{abstract}
SUMMARY
We comment on several topics of the Chilean scientific journals indexed in SciELO (Scientific Electronic Library Online) regarding: manuscript format, article format, review process, and other aspects that could improve their reputation and name and enhance recognition by international journals. Some of these topics are further developed, taking as example Bosque, the most important Chilean forestry journal and the only one that is indexed in SciELO. We suggest to increase the practice of accepting a wider range of manuscript computational formats, to establish an on-line system for submitting manuscripts (currently, $43.3 \%$ of the journals still require hardcopy submissions), to provide a template file for manuscripts to be available online, to streamline the categories of contributions accepted by journals, to establish the blind peer-review process (only $6 \%$ of the journals use it), and to have associate editors. Also, we suggest for Bosque to broaden the institutional diversity of reviewers.
\end{abstract}

Key words: peer-review process, scientific publications, forest research, Chile, SciELO.

\section{RESUMEN}

Se analizan aspectos relacionados con el proceso de publicación de las revistas científicas chilenas seriadas en SciELO (Scientific Electronic Library Online), como el formato y envío de manuscritos, consistencia en la forma de los artículos, proceso de arbitraje, y otros aspectos que podrían ser mejorados para aumentar su estatus y el reconocimiento internacional de revistas científicas. Algunos de estos aspectos son discutidos con mayor profundidad para la revista Bosque, la más importante y la única revista chilena de ciencias forestales indexada en SciELO. Se sugiere ampliar el formato computacional de los manuscritos aceptados por las revistas, establecer un sistema de envío de manuscritos en línea (un 43,3\% de las revistas aún requiere el envío de copias impresas), dejar a disposición un archivo tipo para el envío de manuscritos (ninguna revista ofrece esta alternativa), reducir el número de categorías de tipo de contribuciones aceptadas por las revistas y fusionarlas en una menor cantidad, e implementar mayormente el sistema de arbitraje ciego (sólo un 6\% de las revistas lo emplea) así como la modalidad de editores asociados. Para Bosque en específico, se recomienda además que aumente la diversidad institucional de los evaluadores.

Palabras clave: arbitraje, publicaciones científicas, investigación forestal, Chile, SciELO.

\section{INTRODUCCIÓN}

La divulgación del conocimiento científico mediante publicaciones contribuye al desarrollo de la sociedad. La ciencia ha aportado enormemente al progreso humano y al desarrollo de la sociedad moderna (Annan 2003). La ciencia con mayor impacto es aquella cuyos frutos se transmiten mejor, por lo tanto, comunicar los resultados de ésta es tan importante como la ciencia misma (Clark 2005). Similares llamados al aumento de publicaciones o de su importancia en Chile han sido también planteados para todas las disciplinas en general (Allende et al. 2005), y en específico para las ciencias forestales (Peredo 1991), economía (Edwards 2003) y medicina (Gálvez 2006). Con respecto a esto, y dado que Internet es un elemento vital en el flujo de información a nivel mundial, Chile ha avanzado enormemente con el proyecto SciELO Chile (Scientific Electronic Library Online), el cual, luego de una estricta selección, ha seriado un total de 67 revistas nacionales en un único portal web (http://www.scielo.cl), en donde es posible acceder gratuitamente a los artículos a texto completo (en formato html o pdf). SciELO es desarrollado en 
Chile por la Comisión Nacional de Investigación Científica y Tecnológica (CONICYT). SciELO también se encuentra en Argentina, Brasil, Colombia, Cuba, España y Portugal, y en desarrollo en Costa Rica, México, Paraguay, Perú y Uruguay (SciELO 2007). Las áreas científicas cubiertas por las revistas chilenas de SciELO son las agrícolas, artes, derecho, edafología, economía, forestales, ingeniería, literatura, matemáticas y medicina, entre otras.

Un artículo científico-técnico llega a ser tal luego de un largo y difícil proceso. Para que un artículo sea publicado, un manuscrito (o borrador) debe ser sometido a una revisión realizada por pares (proceso denominado arbitraje), en donde es criticado por investigadores (denominados árbitros o evaluadores) del área temática del manuscrito. El editor de la revista, basado en el arbitraje, decide si el manuscrito tiene los méritos suficientes como para ser publicado. Este proceso de arbitraje le otorga a las publicaciones científicas un alto valor de conocimiento en el tema abordado por éstas, ya que para tal efecto deben sortear la revisión de investigadores (idealmente expertos en esos temas) quienes normalmente abordarían el mismo tema bajo una diferente aproximación, con otras metodologías y que incluso podrían interpretar los resultados de una manera distinta. Por lo tanto, que un manuscrito sea aceptado para publicación, es decir, se transforme en artículo, es un reconocimiento a un trabajo científico-técnico innovador y de interés para la comunidad científica y también en general para cualquier potencial lector interesado.

La revista Bosque, editada desde 1975 por la Facultad de Ciencias Forestales de la Universidad Austral de Chile (UACh), es hoy en día sin lugar a dudas la revista de las ciencias forestales en Chile. Tal como ya lo presagiaba Peredo (1991), las revistas forestales con baja periodicidad y políticas poco claras debieran desaparecer. Peredo (1991) proponía qué esfuerzos deberían conducirse para aunar fuerzas en torno a una única revista científica chilena de las ciencias forestales, dejando fuera el orgullo de las instituciones editoras de las revistas existentes a esa fecha. La visión de Peredo se hizo realidad, no obstante, sin mediar ninguna coordinación interinstitucional, sino que más bien por el peso de la calidad de las publicaciones y manejo de las revistas, algo así como una "selección natural". Actualmente las revistas científico-técnicas forestales chilenas que podrían seguir en calidad a Bosque, a saber las revistas Ciencia e Investigación Forestal (editada desde 1987 por el Instituto Forestal, INFOR) y Ciencias Forestales (editada desde 1978 por la Facultad de Ciencias Forestales de la Universidad de Chile), poseen una baja regularidad en sus números publicados, siendo ésta aún menor que la reportada por Peredo (1991). Esto, sumado a la larga trayectoria y el creciente interés del sector forestal por publicar en Bosque (Gerding 2005), la periodicidad de los números y el aumento de la calidad de sus publicaciones, ha permitido que Bosque sea la única revista forestal chilena indexada en SciELO. Bosque está en SciELO desde el 2002 (volumen 23, número 2), y desde el 2003 publica tres números anualmente, lo cual es causa-efecto de un aumento de los manuscritos enviados dado que es una de las pocas revistas forestales en SciELO a nivel iberoamericano.

Bosque debe seguir mejorando su estatus para llegar a ser reconocida internacionalmente. El buen nivel de la investigación y educación forestal en Chile es bien reconocido a nivel iberoamericano. Así también, la importancia del sector forestal chileno en las exportaciones nacionales, la alta tecnificación en el manejo de plantaciones de Pinus radiata D. Don y Eucalyptus globulus Labill., E. nitens Deane et Maiden, y el manejo de los bosques naturales, posicionan a Chile como un país forestal en Iberoamérica. En este contexto, y teniendo a Bosque como la revista chilena de las ciencias forestales, aunque SciELO le otorga un sello de calidad iberoamericano, las metas próximas de Bosque debieran ser: posicionarse como la revista forestal de mejor calidad de publicaciones en Iberoamérica y finalmente ser reconocida por ISI (Institute for Scientific Information), con lo cual entraría a un concierto internacional más competitivo. A pesar del progreso en cuanto a formato y manejo de la revista en los recientes años, principalmente los últimos dos, algunos aspectos aún pueden elevar su calidad. Estos aspectos también son aplicables a gran parte de las revistas chilenas en SciELO, debido a que éstas presentan formatos y procedimientos similares.

En este trabajo se revisan aspectos generales relacionados al proceso de envío y formato de manuscritos y del equipo editorial para las 67 revistas seriadas en SciELO-Chile (http://www.scielo.cl, consultado el 21 de agosto 2007). Lo descrito en esta opinión está basado exclusivamente en la información entregada por las revistas en el sitio web de SciELO-Chile. Luego, y basándose en la revista Bosque, se discuten aspectos a mejorar tanto del proceso de envío y evaluación de manuscritos, así como también del formato mismo de la revista. Algunos de estos aspectos son comparados con los de otras revistas chilenas. Además, se proponen sugerencias para mejorar los aspectos analizados tanto de la revista Bosque como los de otras revistas chilenas. El objetivo del documento es aportar a la discusión respecto al formato y procesos involucrados en la revista Bosque en particular y en general para las otras revistas científicas nacionales, con el propósito de contribuir a mejorar su calidad y reconocimiento a nivel local e internacional.

\section{FORMATO}

Formato computacional de manuscritos. Con respecto a programas y formatos computacionales del documento, todas las revistas chilenas permiten el envío de manuscritos editados en Microsoft Word. Esto es amigable dado que la mayor parte de la comunidad científica conoce los programas del sistema operativo (S.O.) Microsoft Windows, como lo es M. Word. El problema radica en 
que la inmensa mayoría (97\%) de las revistas exige que los manuscritos sean preparados en M. Word. Esto resulta desalentador para aquellos autores que prefieren trabajar en programas Windows distintos a $\mathrm{M}$. Word y peor aún para aquellos que emplean S.O. como Linux o trabajan en máquinas Macintosh (aunque para estas últimas existe hoy también una versión de Microsoft Office). Si bien tanto en Linux como en Macintosh existen los medios como para transformar hacia formato $\mathrm{rtf}$ (Rich text format), el cual sí es aceptado por la inmensa mayoría de las revistas chilenas, así como también por Bosque, muchas de las bondades de editar artículos en programas distintos a $\mathbf{M}$. Word (por ejemplo, la edición de ecuaciones) pueden ser perdidas o disminuida su calidad en esta transformación de formatos de archivos.

Una solución a esto, al menos en el envío de manuscritos, es permitirlos en formato pdf (Portable document format), vale decir, en un solo archivo puede venir el manuscrito completo, claro está, siguiendo las pautas de cada revista (por ejemplo, con las figuras y cuadros al final). Esta práctica es sólo preferida por tres revistas chilenas: Biological Research, Cuadernos de Economía y Revista Chilena de Historia Natural. El enviar un solo archivo en formato pdf ofrece las siguientes ventajas: a) permite fácilmente mezclar archivos confeccionados en distintos programas, b) se evitan problemas de desconfiguración al abrir un archivo M. Word de una versión distinta en un S.O. Windows más actual (o más antiguo) o incluso en una versión distinta de M. Office, c) el documento luce tal cual como el autor lo quiere y asimismo ocurrirá con todos los evaluadores que vean el manuscrito, d) el tamaño del archivo electrónico será (en gran parte de las ocasiones) menor que enviando las figuras o imágenes y tablas insertas en un documento M. Word o éstas por separado, y e) crear un archivo pdf es gratis si se hace vía Internet (por ejemplo, http://www.pdfonline.com/), así como también se pueden instalar programas en un PC sin necesidad de estar conectados a Internet (por ejemplo, http://www.primopdf.com).

Incluso las revistas podrían despreocuparse del programa computacional empleado en la edición de los manuscritos hasta que éstos hayan sido aceptados o estén pronto a serlo. Es más, mientras éstos cumplan con los estándares de formato de las revistas y sean legibles y entendibles por los evaluadores (al menos en forma) debiera bastar. Tan sólo la Revista Chilena de Anatomía y la Revista de la Ciencia del Suelo y Nutrición Vegetal permiten la edición de manuscritos en Macintosh (además de M. Word), y mejor aún esta última, ofrece también la opción de recibir artículos preparados en formato ASCII. La amplia gama de formatos aceptados por Revista de la Ciencia del Suelo y Nutrición Vegetal es una buena opción a imitar por las otras revistas chilenas.

Ninguna revista chilena acepta manuscritos LaTeX (Lamport 1985), un sistema computacional gratuito de preparación de documentos científicos de alta calidad que ha gozado de popularidad entre un grupo importante de científicos por más de 15 años y que hoy en día es también aceptado en las mejores revistas internacionales. En el caso de las ciencias forestales, por ejemplo, manuscritos preparados en LaTeX son aceptados por prestigiosas revistas como la Canadian Journal of Forest Research, Forest Ecology and Management y Forest Science. Escapa al objetivo del presente artículo detallar las bondades de LaTeX, sin embargo, destaca el hecho de que posee notables ventajas en cuanto a la inserción de figuras y cuadros, edición de expresiones matemáticas y manejo de bibliografía. A estas bondades se suma que un manuscrito preparado en LaTeX es tan sólo un archivo de formato ASCII. En definitiva, existiendo la posibilidad de aceptar manuscritos en formato LaTeX, el abanico de potenciales autores podría ampliarse. Esto último se plantea en el sentido de que cuando un autor debe decidir a qué revista enviar a arbitraje su trabajo, y ante igualdad en calidad de las revistas (y el tiempo promedio de publicación), seguramente optaría por aquella donde el proceso de preparación del manuscrito le sea más fácil.

Formato de figuras. Sólo un 25,4\% de las revistas chilenas, dentro de las cuales no se encuentra Bosque, posee instrucciones específicas, indicando todas o algunas de las siguientes características: resolución, tamaños máximos y formato computacional (jpg, jpeg, bmp, tif, pdf). La mayoría, en cambio, sólo manifiesta que deben estar dentro de un archivo M. Word, el cual usualmente es separado del archivo del texto del manuscrito. Algunas revistas, como Agricultura Técnica y Archivos de Medicina Veterinaria, piden explícitamente que las figuras sean preparadas en Microsoft Excel (así como Investigaciones Marinas requiere cuadros preparados en M. Excel). Aparte de lo complicado que puede ser representar en M. Excel complejos gráficos, la calidad de las figuras en M. Excel está lejos de ser superior a programas que son usados por los autores en sus análisis o bien en programas para estos fines (por ejemplo, SigmaPlot). Se entiende que M. Excel ofrece versatilidad y es en varias ocasiones una buena alternativa para gráficos a ser editados en revistas científicas, sin embargo, exigir esto es injustificado, ya que en programas de ambiente Windows, así como en Linux y en Macintosh, es posible generar figuras de mejor calidad, y lo que es mejor, empleando menos tiempo del autor.

La solución a esto es simple, las revistas tan sólo debieran definir los formatos computacionales de los archivos de las figuras, así como la resolución de éstas, y los autores debieran preocuparse de preparar figuras de un tamaño suficiente como para ser legibles cuando éstas se reduzcan de tamaño en la publicación. Ahora bien, si una revista no posee ningún tipo de requerimiento respecto al formato de las figuras, lo cual puede también entenderse como una ventaja ya que la revista le da libertad absoluta al autor para preparar cualquier tipo de figura, eso debe ser indicado en las instrucciones a los autores. Si los 
formatos o las figuras mismas son compatibles con la producción editorial de cada revista, es algo que también pudiera implementarse. Por ejemplo, el autor, al momento de enviar un manuscrito, podría verificar (en algún sitio web que provea la producción editorial de la revista) si el formato de las figuras cumple con los estándares de la revista. Esta modalidad es implementada, por ejemplo, por la revista Forest Science.

Tipos de contribuciones. Las revistas chilenas son generosas en la clasificación de las contribuciones, existiendo en general los siguientes tipos: artículos, opiniones, notas técnicas, notas científicas, revisión bibliográfica y avance de investigación, entre otras más específicas. Al revisar algunas revistas, existen varias notas que podrían ser perfectamente clasificadas como artículos, e incluso de mayor calidad que algunos artículos. Es claro que existe una gran arbitrariedad en la decisión de clasificar un manuscrito como artículo o nota, y de seguro un tiempo considerable es ocupado por evaluadores y editores en decidir en qué categoría dejar un manuscrito. Dado lo anterior, cabe preguntarse: ¿Cuál es la utilidad de esta segregación? En la práctica tanto una nota como un artículo serán citados de la misma manera y cualquier lector que los vea citados no sabrá su diferencia. El juicio final de la calidad de un artículo (o nota) será dado por el número de artículos que lo cita y en qué revistas está siendo citado. Probablemente ahí se establecería que un artículo es más citado que una nota, sin embargo, eso ya no es tarea de la revista. Entonces, ¿para qué molestarse en clasificar tanto los manuscritos? Esto se justifica cuando las notas son artículos cortos que pueden ser útiles a otros investigadores, y que tardarán un tiempo considerablemente mayor en ser publicados como artículo normal. Bosque afortunadamente, luego del cambio de instrucciones a los autores en 2006, ha reducido considerablemente el número de contribuciones a tres (artículo, nota y opinión).

\section{PROCESOS}

Instrucciones para el envío de manuscritos. Cada revista define sus formatos respecto a secciones, cómo presentar resultados y citar la bibliografía, entre otras. Al respecto, se comentan a continuación algunos temas de lo que hoy en día existe.

Excesiva importancia a los valores- $P$ : mucho del énfasis sobre la interpretación y presentación de resultados estadísticos exigidos por las revistas pasa por los valores- $P$ $(P$-values $)$. Esto hace que gran parte de las conclusiones y análisis en los artículos sean basados casi exclusivamente en el valor- $P$ calculado, no obstante, la estadística ofrece más herramientas que la reducción de toda una investigación a un solo número, como el valor- $P$. La estadística debe ser considerada como una ayuda para extraer qué están "diciendo" los datos y no como una herramienta que diga lo que se debiera hacer. El énfasis en las instrucciones de las revistas respecto al uso de estadística debiera ser simplemente enfocado a que los artículos entreguen al menos medidas claras de variabilidad de sus datos (por ejemplo, a través de errores de estimación, coeficientes de variación, errores de muestreo, entre otros). Tampoco es bueno restringir y acotar demasiado las instrucciones en estadística, ya que esta disciplina (así como otras) avanza rápidamente y novedosas (o al menos poco tradicionales) aproximaciones, como por ejemplo la estadística bayesiana, quedarían fuera. Si bien aquí no se presenta un estudio respecto al nivel de los análisis estadísticos de las revistas chilenas, el nivel mostrado, al menos en general por los artículos publicados en Bosque, parece ser satisfactorio. No obstante, Torres et al. (2005), al realizar un estudio de los análisis estadísticos en revistas científicas a nivel latinoamericano (incluyendo Chile), encontraron varias anomalías. A este respecto, futuros estudios podrían llevarse a cabo en las revistas chilenas.

Estandarización de abreviaturas de revistas: todas las revistas chilenas presentan un formato respecto a cómo citar la bibliografía. Este punto, aunque diferente entre revistas, significa una inversión importante del tiempo necesario para el envío de un manuscrito. $\mathrm{Al}$ respecto, podrían adoptarse algunas estandarizaciones, al menos entre grupos de revistas de similares temáticas (las médicas, las de los recursos naturales, etc.), para que el cambio de formato para los autores no sea tan drástico. Ninguna revista chilena presenta una estandarización de abreviaturas de los nombres de las revistas. La ausencia de una estandarización de este tipo provoca inconsistencia en la forma de la revista. Por ejemplo, en Bosque es frecuente encontrar en algunos artículos citando el Journal of Biogeography como J. of Biogeography y en otros empleando el nombre completo, lo cual también ocurre con otras revistas (New Zealand Journal of Botany). Una estandarización reduciría el número de palabras (y letras) de los artículos significativamente, por ejemplo, Forest Ecology and Management podría ser reducido a For. Ecol. Mana., Canadian Journal of Forest Research a Can. J. For. Res., y así también con otros. Referencias para abreviaciones pueden ser obtenidas de revistas ISI (en el caso forestal, la propuesta por la Society of American Foresters). Siendo aún más ambiciosos, y ya que todas las revistas aquí analizadas están bajo el alero de SciELO-Chile, cada revista podría proponer un listado de abreviaciones de las revistas comúnmente citadas en ellas, luego éstas se incluyen en un gran listado a nivel nacional, en donde previamente se discuten aquellas que son comunes. Más aún, esto podría luego ser expandido a nivel latinoamericano, nuevamente gracias a la estructura y coordinación de SciELO.

Archivo plantilla: ninguna revista ofrece una plantilla con el formato requerido para el envío de manuscritos. Varias, si no todas las instrucciones brindadas por cada revista, podrían ser mejor explicadas a través de un archivo tipo o plantilla (template), el cual podría ser empleado por cada 
autor que piensa enviar un manuscrito. Este archivo (ya sea en formato M. Word, para Macintosh u otro) debiera quedar disponible en el sitio web de la revista. Lo mismo podría hacerse con cuadros y figuras. Con respecto a estas últimas, la Revista Chilena de Infectología es la única que ofrece ejemplos claros de las figuras que se requieren. Nótese que este archivo es sólo una ayuda que debiera facilitar tanto el envío como el arbitraje de los manuscritos. En otras revistas internacionales no se dan indicaciones, ya que existe una mayor "cultura de publicación" de los investigadores; sin embargo, se cree necesario una mayor estandarización para Chile y en general para Latinoamérica, dada la baja cultura de publicación.

Tipo de arbitraje. Sólo cuatro revistas (6\%) (Agricultura Técnica, Cuadernos de Economía, Electronic Journal of Biotechnology y EURE) emplean el sistema de "arbitraje ciego", en donde tanto los evaluadores como los autores de los manuscritos permanecen anónimos durante el proceso de evaluación. Este sistema de arbitraje garantiza una mayor imparcialidad de la decisión. En cambio, la inmensa mayoría de revistas emplea el sistema de "árbitros desconocidos", en donde el nombre de los evaluadores no es revelado a los autores, pero los evaluadores conocen la completa identificación de los autores del manuscrito sometido a revisión. El sistema de arbitraje ciego debiera ser mayormente adoptado por las revistas chilenas, ya que no tan sólo ofrece mayor imparcialidad, sino que basado en el bajo número de investigadores en Chile (Allende et al. 2005) resulta fundamental evitar al máximo celos interinstitucionales o intergrupales de trabajo. También podría existir la opción de que un evaluador indique si prefiere o no ser identificado y aparecer, por lo tanto, en la evaluación del manuscrito que el autor recibirá.

Envío de manuscritos. Casi la mitad de las revistas chilenas $(43,3 \%)$ aún requiere que los autores envíen copia(s) impresa(s) de sus manuscritos, lo cual retrasa el proceso de arbitraje. Por otra parte, aunque gran parte de las revistas permite el envío de manuscritos vía correo electrónico (e-mail), ninguna posee un sistema en línea (on-line) de envío de manuscritos (como por ejemplo, los implementados por las revistas del grupo Elsevier). Un sistema de este tipo podría encargarse de compilar el manuscrito (para un gran variedad de formatos) en el formato preciso para el manejo del editor y de los evaluadores, o bien tan sólo recibir el archivo pdf, como fue sugerido anteriormente. Esto también ayudaría al seguimiento del manuscrito por parte de los autores, de los evaluadores y del editor. Un sistema de envío de manuscritos en línea también favorece la oportunidad de contar con editores y evaluadores extranjeros.

Otra ventaja de un sistema de envío y revisión de manuscritos en línea, es que ayudaría a la implementación de una sección de artículos In-Press, dentro del portal donde están a disposición los artículos. Estos son manuscritos ya aceptados, que están en un formato editado por la producción editorial y gráfica de la revista, por lo tanto con la apariencia casi definitiva que tendrán cuando sean finalmente publicados, aunque sin número de volumen ni de páginas. Esto favorece la revisión actual de trabajos por parte de investigadores que inician estudios similares. Así también, dejaría algo más conformes a los autores descontentos por la larga espera en la publicación de sus artículos. La revista Cuadernos de Economía implementa esta opción en su sitio web (pero no en su portal en SciELO).

\section{LLEVANDO BOSQUE A UN NIVEL SUPERIOR}

Si bien Bosque pertenece al grupo SciELO, aún queda un gran paso para que realmente se inserte en el concierto de las publicaciones internacionales. Sólo como referencia, de acuerdo a SciELO (2007) el factor de impacto de Bosque en el 2006 fue 0,0481, mientras que para Forest Ecology and Management y Forest Science fueron, según Web of Science (2007), 1,839 y 1,457, respectivamente. Si bien este indicador puede no ser el mejor y otros indicadores del impacto de las revistas pueden ser derivados (como los calculados por Contreras et al. (2006)), estos valores muestran una amplia brecha de diferencia. Similar situación ocurre con las revistas en el área biomédica (Gálvez 2006). En este contexto, el ingreso de Bosque a la categoría ISI resultaría un aliciente, aunque una ardua tarea, considerando que tan sólo siete de las 67 revistas chilenas (Archivos de Medicina Veterinaria, Biological Research, Electronic Journal of Biotechnology, Journal of the Chilean Society, Revista Chilena de Historia Natural, Revista Geológica de Chile y la Revista Médica de Chile) en SciELO están indexadas en ISI (Web of Science 2007). Para llevar a Bosque a un nivel superior al que actualmente sustenta y plantearse ser la revista más importante y prestigiosa de las ciencias forestales en Iberoamérica, debe difundir y abrir mayormente sus puertas a trabajos extranjeros, así como experimentar una mejora en: a) la consistencia en el formato de los artículos publicados, b) el aumento de la cantidad y apertura del espectro de evaluadores y c) implementación de una modalidad de editores asociados.

- Consistencia en el formato de los artículos publicados: el formato de presentación de un artículo es un aspecto central dentro de la forma de una revista científica. De esta manera, la presentación de un artículo se estandariza independientemente del tema abordado, siendo consistente. Bosque ha mejorado considerablemente este aspecto en los últimos dos años, y tan solo se destaca este punto para reforzar la idea de mantención del formato por al menos algunos años. Una inconsistencia relativamente frecuente de algunos de los artículos publicados en Bosque previos al 2006, es referente a la dirección de los autores. En varios artículos es común ver que los autores 
indicaban, además de la dirección postal, sus grados académicos o títulos profesionales. Requerir la institución y dirección postal de los autores, y para el autor de correspondencia además solicitar el correo electrónico, teléfono y fax debiera ser suficiente.

- Aumentar la cantidad y abrir el espectro de evaluadores: Bosque publica el comité científico de la revista, actualmente integrado por 46 investigadores nacionales e internacionales $(23,9 \%)$, el cual en los últimos siete años no ha variado mayormente. La labor de este comité no es especificada en Bosque, aunque lo es en muchas otras revistas chilenas; sin embargo, se asume que el comité científico no es necesariamente el evaluador de los manuscritos enviados a Bosque, y son otros también los evaluadores.

Una medida que ayuda a transparentar el proceso de arbitraje es publicar el listado de los evaluadores de trabajos de un número o en un año completo. Desgraciadamente, tan sólo tres revistas han establecido esta práctica el 2006. La Revista Musical Chilena tiene disponible el listado de los evaluadores del último número publicado en su sitio web en SciELO. Mejor aún, la Revista Chilena de Historia Natural publicó en el último número de 2006 (volumen 79, $\mathrm{N}^{\circ}$ 4) los evaluadores de ese año (así también en el 2002). Siguiendo esta buena práctica, Bosque ha dejado a disposición (volumen 28, $\mathrm{N}^{\circ}$ 1) el listado de los evaluadores de los artículos publicados en el 2006. La publicación de este tipo de listado, además de transparentar el proceso de arbitraje, es un merecido reconocimiento a los árbitros que noblemente disponen de su tiempo en forma gratuita para evaluar manuscritos.

El listado publicado por Bosque corresponde al de los 84 evaluadores que colaboraron como árbitros de los manuscritos de la revista durante el año 2006 y para el volumen 27. De aquí se deriva que estos árbitros participaron en la evaluación de los artículos efectivamente publicados en el volumen 27 (el cual corresponde al año 2006) así como también de una fracción de los que serán publicados el 2007 (y quizás el 2008). Aquí sólo se asumirá que estos evaluadores tienen injerencia sobre los 26 artículos que fueron publicados por Bosque en sus tres números del volumen 27. Al analizar este listado, es posible establecer que: a) el $44 \%$ de los árbitros está adscrito a la UACh o a unidades dependientes de ella, b) sólo un 10,7\% es evaluador extranjero, c) tan sólo un $28,3 \%$ del comité científico evaluó manuscritos (de este porcentaje un $69,2 \%$ es investigador UACh y ninguno es extranjero), d) siendo conservador, y considerando que el $100 \%$ de los manuscritos enviados fueron publicados, implica que cada artículo fue evaluado, en promedio, por entre dos (el mínimo establecido en la normativa de la revista) y tres árbitros. Dada la alta proporción de evaluadores UACh, según estos datos, uno de los evaluadores debiera pertenecer a esta institución.
Si bien no se duda del profesionalismo de la evaluación de los árbitros UACh, estos datos son desalentadores para aquellos autores que plantean trabajos con ideas distintas y quizás muy opuestas a las que algunos investigadores UACh sostienen. Además, es una sana práctica en revistas científicas el ampliar la evaluación a diferentes aproximaciones y enfoques a similares temáticas. Por lo tanto, es recomendable que Bosque amplíe el espectro institucional de los evaluadores. Esta ampliación debe ser tanto a nivel nacional como internacional, contando con una mayor presencia de evaluadores nacionales de otras instituciones, extranjeros, chilenos trabajando en el extranjero, o bien investigadores reconocidos internacionalmente en un área temática.

- Implementar la modalidad de editores asociados: un 13,4\% de las revistas chilenas ofrece esta modalidad; sin embargo, sólo la Revista Chilena de Infectología la implementa detalladamente, indicando el área temática de cada editor(es) asociado(s). Esto permite una mayor agilidad en el proceso de revisión, ya que el editor puede fiarse mayormente en la expertise de los editores asociados para una selección más idónea de los mejores evaluadores para cada manuscrito enviado. Tentativamente podría designarse más de un editor asociado por área dependiendo de la oferta de manuscritos en los últimos años. Algunas áreas que se vislumbran en Bosque podrían ser: biometría, biotecnología, botánica, cosecha, edafología, economía, hidrología (cuencas hidrográficas), silvicultura, sensores remotos y sistemas de información geográfica y productos forestales. Dependiendo de la difusión de Bosque hacia países con bosques tropicales, áreas como silvicultura tropical debieran también ser incorporadas.

\section{DISCUSIÓN}

Cada revista debe buscar el balance óptimo entre preparar instrucciones para autores con mayor o menor detalle. Las mejores revistas científicas internacionales suelen tener instrucciones generales, y exigiendo básicamente enviar manuscritos que cumplan con normas universales de escritura científica. Existen dos razones que justifican esto. Por una parte, los autores poseen cultura de publicación en revistas científicas, por lo tanto son ellos mismos los que saben preparar un documento lo suficientemente claro y ordenado, y, por otra, dichas revistas cuentan con personal especializado en la edición de revistas científicas, lo cual ayuda al manejo más eficiente de manuscritos, así como a mejorar detalles de formato de los manuscritos aceptados. Un escenario opuesto es el de las revistas SciELO chilenas no ISI y en especial de Bosque. Primero, los autores nacionales poseen una baja tasa de publicación en revistas científicas, por lo tanto una escasa experiencia en preparación de publicaciones; esto implica que el proceso de revisión se hará más lento 
debido a que tanto editores como árbitros deberán ocupar gran parte de su tiempo en detalles de forma en lugar de centrarse en aspectos de fondo. Dada esta situación, se cree apropiado que las revistas chilenas (y Bosque) pongan bastante atención en el diseño de las instrucciones para autores. Segundo, estas revistas se editan mayormente en base a esfuerzo de entusiastas, apoyados por subvenciones, subsidios y voluntariado de personas, pero sin personal especializado en edición de revistas. Sin lugar a dudas, para que revistas nacionales puedan acceder a ISI deben contar con apoyo profesional en edición de revistas. Es de esperar que el Gobierno de Chile contribuya mayormente a financiar lo anterior de alguna forma (mediante CONICYT), por ejemplo seleccionando a las mejores revistas dentro de SciELO por disciplina.

Chile posee una masa importante de potenciales autores de artículos, lo que sumado mayormente al aporte latinoamericano y de otras regiones implica que es posible mantener revistas de prestigio. Para el caso forestal, Peredo (1991) criticaba al sector por falta de tradición y costumbre de publicar regularmente en revistas científicas. Aquello correspondía a la situación del sector en ese tiempo, en donde, por una parte, existía una alta demanda del mercado por ingenieros forestales, que mayoritariamente se desempeñaban en empresas, las cuales no buscan difundir conocimiento científico. Por otra parte, en el mundo académico, aunque existían profesores con formación de postgrado, las exigencias universitarias para ellos no eran las mismas de hoy, en donde publicar es parte fundamental de la labor de un académico universitario. Así también, hoy existe una mayor masa de profesionales con grados académicos de master y doctorado, por lo tanto con una mejor formación para publicar. Esto debiera hacer que el nivel de potenciales publicaciones aumente en calidad así como en cantidad. Esto último, potenciado de igual forma por el creciente número de postgrados forestales y no-forestales ofrecidos en Chile, los cuales también están atrayendo estudiantes latinoamericanos. Todo esto hace pensar que Bosque (así como la mayoría de las revistas científicas chilenas) debiera tener una cantidad suficiente de manuscritos de calidad con los cuales avanzar hacia una categorización ISI.

Tal como fue planteado por Gerding (2005), el futuro de Bosque es promisorio, sin embargo, nuevas mejoras pueden aún ser aplicadas, manteniendo la consistencia en la forma de los artículos publicados. Es de esperar que los llamados a aunar esfuerzos para seguir potenciando Bosque, planteados por Peredo (1991), Gerding (2005) y en este artículo, sean recibidos por los investigadores de las ciencias forestales en Chile. Potenciar una sola revista científica forestal debiera contribuir mayormente a construir una cultura de investigación. Lamentablemente, iniciativas recientes (2007) como la reedición de la revista Ciencia e Investigación Forestal del INFOR, luego de nueve años sin números, hace pensar que las lecciones del pasado respecto al número de revistas científicas forestales de buena calidad que puede sostener Chile no han sido completamente aprendidas. Es posible que aún no sea el momento en que la comunidad científica nacional pueda sostener más de una revista científica forestal; por el contrario, los esfuerzos para mejorar Bosque deben seguir una planificación en torno a buscar su categorización ISI. De todas maneras, como lo planteado por Salas (2007), revistas con un enfoque mayormente técnico (en donde estarían Ciencia e Investigación Forestal, Ciencias Forestales, Bosque Nativo y otras) son necesarias para el desarrollo de un país.

Bosque, así como otras revistas científicas chilenas interesadas en llegar a ser reconocidas por ISI, deben abrirse aún más a recoger evaluadores latinoamericanos así como también manuscritos extranjeros. Aunque las grandes diferencias de inversión en investigación científica en Latinoamérica versus Norteamérica y Europa (CONICYT 2004) indican una clara desventaja en el número de publicaciones científicas latinoamericanas, Holmgren y Schnitzer (2004) demostraron que la productividad en publicaciones ISI (medida de acuerdo a algunos indicadores) en Latinoamérica ha aumentado considerablemente en los últimos diez años, incluso a mayores tasas que en Norteamérica y Europa. Esto nuevamente demuestra que debiera existir la oferta suficiente de investigadores de primer nivel que puedan no tan solo evaluar trabajos sino que también enviar manuscritos.

\section{CONCLUSIONES}

Las revistas científicas chilenas podrían realizar mayores esfuerzos tendientes a ampliar el formato computacional de los manuscritos aceptados por las revistas, establecer un sistema de envío de manuscritos en línea, dejar a disposición un archivo tipo para el envío de manuscritos, reducir el número de categorías de tipo de contribuciones aceptadas por las revistas, e implementar mayormente el sistema de arbitraje ciego así como la modalidad de editores asociados.

El manejo editorial de Bosque ha mostrado una clara mejora en el último tiempo, sobre todo en los últimos dos años. De todas maneras, y para continuar mejorando Bosque, podría implementar los cambios mencionados anteriormente y además ampliar el espectro institucional de los evaluadores.

\section{AGRADECIMIENTOS}

A Gabriel Mancilla (Universidad de Chile), Patricio Núñez (Universidad de La Frontera), Claudio Donoso (Universidad Austral de Chile), Florencia Montagnini y Juan Eberhard (Yale University, USA) por sus comentarios en un primer borrador del presente trabajo. Las detalladas observaciones de los evaluadores ayudaron a mejorar este trabajo. Cualquier error remanente en el artículo es responsabilidad del autor. 


\section{REFERENCIAS}

Allende JE, J Babul, S Martínez, T Ureta. 2005. Análisis y proyecciones de la ciencia chilena 2005. Academia Chilena de Ciencias - Programa Bicentenario de Ciencia y Tecnología CONICYT, Santiago, Chile. 427 p.

Annan K. 2003. A challenge to the world's scientists. Science 299(5612): 1485.

Clark ML. 2005. El ISI reconoce el impacto de nuestra revista. Revista Panamericana de Salud Pública 17(2): 73-74.

CONICYT (Comisión Nacional de Investigación Científica y Tecnológica, CL). 2004. Gasto en I+D como proporción del PIB en Chile y otras economías seleccionadas, año 2004. Comisión Nacional de Investigación Científica y Tecnológica, Gobierno de Chile. Consultado 4 septiembre 2007. Disponible en http://www.conicyt.cl/573/article3963.html.

Contreras C, G Edwards, A Mizala. 2006. La productividad científica de economía y administración en Chile. Un análisis comparativo. Cuadernos de Economía 43(128): 331-354.

Edwards S. 2003. Latin American and U.S. universities: A forty years analysis. Cuadernos de Economía 40(121): 399-412.
Gálvez M. 2006. Publicaciones biomédicas: realidad de Chile y Latinoamérica. Revista Chilena de Radiología 12(3): 113-117.

Gerding V. 2005. La trayectoria de Bosque 1975-2005 y su proyección. Bosque 26(3): 3-4.

Holmgren M, SA Schnitzer. 2004. Science on the rise in developing countries. PLoS Biology 2(1): 10-13.

Lamport L. 1985. LaTeX-A document preparation system-User's guide and reference manual. Addison-Wesley, Reading, Massachusetts, USA.

Peredo H. 1991. Revistas científicas del sector forestal chileno ¿otra súplica por menor cantidad y mayor calidad? Bosque 12(1): 11-15.

Salas C. 2007. ¿Dónde va a parar la investigación forestal chilena de "excelencia"?: Pasado, presente y futuro. Bosque Nativo (En prensa).

SciELO. 2007. Scientific electronic library online. Consultado 21 agosto 2007. Disponible en http://www.scielo.org.

Torres V, R Herrera, L Sarduy. 2005. El arbitraje estadístico y su influencia en la calidad de las publicaciones científicas. Revista Facultad de Ingeniería-Universidad de Tarapacá 13(2): 85-89.

Web of Science. 2007. ISI web of science. Consultado 23 agosto 2007. Disponible en http://scientific.thomson.com/products/wos. 